\title{
CVS Health and the Imaginary Worlds of the Institute for Clinical and Economic Review (ICER)
}

Paul C Langley, PhD

Adjunct Professor, College of Pharmacy, University of Minnesota

\begin{abstract}
In August 2018, CVS Health released a position paper detailing policies in place and those being implemented to help reduce the costs of drugs. This paper introduced three new strategies for reducing costs. These are (i) zero out of pocket costs for chronic disease through a preventive drug list; (ii) reducing the launch price through adoption of modeled cost-per-QALY outcomes by the Institute for Clinical and Economic Review (ICER) to guide clients to exclude drugs launched at a price of greater than \$100,000 per QALY; and (iii) introducing tools to be used by doctors, pharmacists and consumers to create greater transparency in understanding the real cost of drugs. The purpose of this commentary is to consider the second of these strategies, the application of a willingness to pay threshold as a viable strategy for impacting launch costs. The arguments presented here are that while modeled cost-per-QALY claims are a staple of formulary committee deliberations in many single payer health systems, their adoption by CVS Health fails to take into account not only the imaginary nature of the modeled construct utilized to generate the cost-per-QALY estimate and its shortcomings but the fact that alternative model structures may render invalid the application of willingness-to-pay thresholds. The case is made that CVS Health could adopt a more rigorous process of formulary assessment to support both preliminary assessments of new products and also an ongoing process of formulary review that challenge manufacturers to justify pricing over product patent life. This process should capitalize on the development of formulary evaluation platforms, potentially involving blockchain technology and smart contracting, for therapy interventions in targeted patient populations. Introducing a more rigorous formulary process, in particular the requirement for claims evaluation protocols, will not only assist CVS Health in restraining price increases over the life of the product but, for the first time in the US, put manufacturers on notice that patently unreasonable pricing policies and claims for product performance can be systematically and effectively challenged.
\end{abstract}

Keywords: CVS Health, Caremark, ICER, pseudoscience, economic evaluations, imaginary worlds, blockchains

\section{Introduction}

Over the past 2 years a number of commentaries have appeared in INNOVATIONS in Pharmacy that have made the case (i) that modeled claims for cost-effectiveness, notably those that rely on lifetime cost-per-quality adjusted life year (QALY) estimates lack scientific merit and (ii) that claims for cost-effectiveness and pricing recommendations based on willingness-to-pay thresholds put forward by the Institute for Clinical and Economic Review (ICER) should be put to one side in favor of the evaluation of modeled claims that are credible, evaluable and replicable ${ }^{1,2}$. Unfortunately, this message has yet to resonate fully with formulary committees and pharmaceutical manufacturers. Among the former would appear to be CVS Health in their latest announcement that as one of the tools at their disposal for reducing overall medical costs, notably the cost of pharmaceuticals, they propose to introduce the rule that allows their clients 'to exclude any drug launched at a price of greater than $\$ 100,000$ per QALY from their plan'3. The choice of product will be based on 'publicly available analyses' from the ICER.

Corresponding author: Paul C Langley, PhD

Adjunct Professor

College of Pharmacy University of Minnesota

Director, Maimon Research LLC

5061 North Apache Hills Trail, Tucson, AZ 85750

Email: langley@maimonresearch.com
While CVS Health believes that the implementation of a $\$ 100,000$ willingness-to-pay cutoff will be a significant addition to their armamentarium, this belief rests on an assumption that lifetime cost-per-QALY claims or similar ICER models have scientific merit. This presumption is far from the case. ICER and similar models lack scientific credibility because the claims made, whether they are for comparative clinical effectiveness or cost-effectiveness are not credible ${ }^{4}$.

The purpose of this commentary is to point to: (i) the limitations of the ICER modeling framework; (ii) the implications of CVS Health factoring in ICER modeled claims with cost-per-QALY thresholds into formulary decisions; and (iii) the need for guidelines for formulary evaluation that have the potential to provide, not only a robust evidence base for evaluating claims but one that can extend over the patent life of products.

\section{Policies Towards Drug Price Inflation}

CVS Health has implemented a number of policies directed towards containing drug prices and costs. CVS Health claims that these initiatives have had a positive and significant impact on adherence and overall health costs ${ }^{3}$. These initiatives are: (i) encouraging the greater use of generics; (ii) application of prior authorizations for effective use; and (iii) strategies to obtain rebates and discounts from manufacturers. Most recently, three further initiatives have been detailed. These are: (i) a preventive drug list for zero out-of-pocket costs for chronic 
disease; (ii) reducing the launch price through comparative effectiveness exclusions; and (iii) the introduction of tools to ensure greater transparency to encourage doctors, pharmacists and consumers to engage in cost control.

Consider the second of these recent initiatives: the program to exclude drugs launched at a price greater than $\$ 100,000$ per QALY from a client's plan. This program rests on the application of ICER modeled claims for cost-effectiveness. If ICER has concluded that, based upon its modeling framework, a 'me to' medication is not cost-effective then the CVS Health program will allow clients to exclude that drug from their plan. This potential for exclusion is a seen as a red flag for manufacturers proposing a launch price that generates an ICER modeled costper QALY greater than a willingness-to-pay of $\$ 100,000$ per QALY. Whether this policy is sustainable is another question.

\section{The ICER Modeling Framework}

The ICER modeling framework has been reviewed in a number of earlier commentaries. Briefly, it is a US version of the National Institute for Health and Care Excellence (NICE) lifetime cost-per-QALY reference case model, versions of which have been introduced by other single payer health systems such as Australia, Canada, Ireland and New Zealand ${ }^{5,6,7,8,9}$. The reference case in the UK mandates that all submissions for new products must be presented, with few exceptions, within a constructed lifetime cost-per-QALY framework. The model is 'for information only' and not, as clearly stated in the latest Canadian guidelines, intended to conform to the standards of normal science: Economic evaluation are designed to inform decisions. As such they are distinct from conventional research activities, which are designed to test hypotheses ${ }^{10,11}$. In short, we should base formulary decisions on 'information' created from imaginary constructs. We are asked to accept that the construct, which often extends decades into the future, is 'informative' because its structure and assumptions are 'reasonable' and 'realistic'. The fact that it is entirely possible, and probably a necessary marketing counterpoint, to build competing 'informative' models is, presumably, beside the point.

One reason for building a lifetime model is that it supports the application of willingness to pay thresholds. Outcomes are expressed as 'all embracing' QALYS to capture morbidity and mortality with assumptions as to lifetime direct medical costs. Drug costs are at the discretion of the manufacturer or a model builder such as ICER, although interestingly enough, ICER makes no assumptions as to how drug prices are expected to increase over the patent lifetime. If they did, modeled costs would be substantially inflated.

Once lifetime QALYs and costs are calibrated, these are expressed as lifetime cost-per-QALYs and matched to thresholds. The favorite thresholds are $\$ 50,000, \$ 100,000$ and $\$ 150,000$ per QALY. ICER uses these thresholds to support pricing recommendations. Obviously, if a manufacturer is preparing a reference case submission, their constructed imaginary world can either be designed to support a base pricing point as part of a long-term incremental pricing strategy or it can be used as a framework for reviewing alternative pricing options. They could even argue for imaginary cost-perQALY sliding scales in $\$ 10,000$ increments depending on the disease state. Indeed, a cynical reviewer might add that a model can support a 'bait and switch' pricing strategy where a deliberately low base price is chosen to ensure that an acceptable threshold is met. Once an acceptable formulary position is agreed, which might even involve short-term discounting to smooth product acceptance; this can then be followed within a matter of months by a succession of incremental price increases.

Consider as an example a new drug which has a proposed annual cost of $\$ 20,000$ at market entry. A succession of annual price increases of $8 \%$ means that in 15 years the cost will have risen to $\$ 54,200$ with further increases until it loses patent protection or is replaced. The fact that manufacturers can exploit their position as a monopolist to extract substantial and increasing rents over the lifetime of a product means that claims that a product is cost-effective are meaningless. The $\$ 100,000$ per QALY willingness-to-pay threshold has no relevance. Different imaginary models will generate different claims.

Previous commentaries have addressed the model shortcomings of a number if ICER product reviews ${ }^{12,13,14,15}$. All have pointed to the often limited basis for modeled assumptions in terms of not only the clinical claims but the translation of these into QALYs. Further limitations noted have been assumptions (or their absence) regarding patterns of adherence and compliance together with, as noted above, assumptions as to the anticipated lifetime pricing of drugs and other direct medical costs. Pointing to these limitations only goes to emphasize the basic weakness of any 'for information' lifetime or long-term modeled claim: it can always be challenged with competing models constructed to come to different conclusions on what may appear to be quite reasonable and well-argued assumptions.

Consider as an example two models. One model, where a target cohort of patients is modeled over their projected lifetimes and which takes account of adherence and persistence with therapy. If compliance is only short term with, say, $50 \%$ of patients initiated to a therapy for a chronic disease discontinuing therapy within two years, then cost-per- QALY claims will apply only to the cumulative and diminishing number of patients assumed to remain on therapy. The competing model, rather than considering just an initial cohort of patients modeled over their lifetimes might take the perspective of patients being recruited by the manufacturer to offset those lost to treatment as well as capturing increased 
market share for a fixed projection period. Over the timeframe of the model new patients will enter each year who will then begin to discontinue therapy. The profile of QALYs will be quite different. If QALYs are time dependent, then the overall QALY profile will be the sum of the QALYs experienced in that year across the cohorts of patients initiated to therapy in each year. Cost-per-QALY claims and the aggregate QALY and cost profile will be different between the two models. Claims for the first model will rest on the outcomes for a modeled cohort of patients initiated to therapy while the second is based on the assumption of a constant or growing cohort of patients with those discontinuing being continuously replaced over the lifetime of the product.

It is also worth noting that there is an ongoing debate over the transparency of the ICER modeling approach and the scope given to manufacturers to challenge the model in the early stages of its development by groups contracted to ICER ${ }^{16}$. A key point of contention is early access to the actual model that would allow manufacturers to challenge the model structure and modify or replace assumptions. While this may appear a somewhat fruitless exercise as the net result could be an 'imaginary worlds challenge cup' (which has been a feature of diabetes modeling) the bottom line is that it serves as a smokescreen ${ }^{17}$. As formulary review groups would typically not have the skills to evaluate the technical and evidence-based attributes of competing models, the result may simply be to put the models to one side in favor of more direct negotiations.

\section{Abandoning ICER for PROST}

If the ICER approach to pricing recommendations is seen as a dead-end, is there an alternative to the construction of imaginary cost-per-QALY worlds and willingness-to-pay thresholds? The answer is 'yes' once we put aside the 'need' for reference cases and focus on the evidence base necessary for initial and ongoing disease area and therapeutic class reviews by formulary committees.

A manufacturer cannot expect a formulary decision to accept a product at a base price and tier position to be unchallenged over the patent life of the product. A manufacturer should recognize that in the case of both an initial formulary decision and future disease area and therapeutic class reviews, it must assure the formulary committee that the claims made initially in respect of comparative clinical benefits are not only credible, evaluable and replicable but that these should continue to be addressed over the patent life. The focus must switch from pseudoscientific constructed 'information' paradigm to one in which 'evidence' drives decisions.

The theme that product claims, which would include both extrapolated and modeled claims, should be credible is addressed in the proposed Minnesota guidelines for formulary evaluations. Version 2.0 of these guidelines released in December 2016 are focused on the evidentiary standards required to support initial informed drug choice ${ }^{18,19}$. Following from the standards established in randomized controlled trials, the proposal is that formulary submissions be accompanied by a study protocol (the PROST protocol) detailing how the claims for clinical outcomes, cost-effectiveness and budget impact are to be evaluated. This evaluation process is relevant both for new products seeking formulary approval as well as for ongoing disease area and therapeutic class reviews. The protocol is intended to support a short-term assessment of claims made with feedback to the formulary committee within a meaningful timeframe. Until those results are available any initial decision on pricing and tier position (including prior-authorization) should be considered provisional. It is proposed that until claims are verified a low provisional price would be agreed. Indeed, even after a final price is agreed following validation, which may be 12 to 20 month later, it is suggested that the manufacturer agree to a fixed price unless a price increase can be justified.

This emphasis on evaluation and replication reflects, as noted above, the limited evidence base supporting all too many clinical claims and the apparent difficulties that independent assessors have had in replicating phase 3 protocol based claims. Claims which are, all too frequently, captured by ICER-type models with no thought given to undertaking an in-house assessment to replicate those claims in the target patient population before launching into construction of an imaginary world.

\section{A PROST Platform}

There are a number of options open to manufacturers submitting a protocol for claims assessment in the choice of platform. Clearly, two of the key requirements for a PROST platform are that they allow physicians and patients to engage with each other and that there is feedback in real time to monitor response to therapy. Other required features, and this will be likely a prospective observational study design, are to ensure randomization within the target population and then ensure that the power of the study can overcome the lack of power of for secondary endpoints that typically characterize RCTs. The fact that underpowered secondary endpoints (including QALYs) are used as the basis for lifetime modeled claim assumptions needs to be addressed. In practical terms, there should be at least a sub-group within those selected to capture the inclusion/exclusion criteria established in the key phase 3 RCTs. To minimize delays in formulary agreement with the manufacturer on a protocol, the draft protocol, to include the proposed evidence platform should be, as outlined in the PROST framework, part of the formulary submission.

One option for the platform is a target population registry. While this is feasible, a more attractive option would be a blockchain ${ }^{20}$. The blockchain has three advantages: first, it is a secure, encrypted and portable transaction ledger; second, it can support medical records which can be linked to any number 
of registries held by the blockchain for initial and ongoing formulary evaluations; and third, it can support incentivized smart contracting by patients to monetize their health data. These aspects of a blockchain as a medical record to support health technology assessments including formulary evaluations have been explored in recent commentaries ${ }^{21,22}$. The overriding consideration, however, is that the ability to construct lifetime health record blockchains puts to one side reliance on driving formulary decisions through the construction of imaginary worlds; an unfortunate attribute of health technology assessment for the past 30 years.

\section{Conclusions}

If CVS Health wishes to factor into their formulary decisionmaking claims for cost-per-QALY outcomes based on the construction of imaginary worlds, they are entitled to do so. However, in embracing the ICER approach the concern is that few people, including probably those making decisions within CVS Health, will have the skills to recognize the inappropriateness and insubstantial nature of the ICER methodology. Taking ICER claims at face value is accepting a black box. If there is one thing we have learned from countries that have a transparent (UK) or pseudo-transparent (Australia) modeling assessment process, is the obvious fact that any model can be constructed or re-engineered to support a range of pricing and outcomes assumptions. If we add to this another obvious fact that expressing outcomes over the lifetime of the patient population or the natural course of the disease asks the reader to accept that we have a crystal ball that enables us to create a realistic world stretching decades into the future with
QALYs and costs accumulating over that timeframe. We are asked to accept the credibility of these claims, or rather the assumptions supporting these claims when we know they were never intended to be subjected to the evidentiary standards of normal science. In fact, they are designed to do exactly the opposite. This reduces to a debate over visions of the future.

To end on a more positive note; with resources available it would surely not be beyond the bounds of possibility for CVS Health to support a commitment to the scientific method in funding a health technology assessment group for assessing claims with competing pharmaceutical products. Certainly, such a commitment would mean rejecting imaginary constructs in favor of an evidence or outcomes based formulary. After all, denying coverage (i.e., access) to a drug on the basis of an imaginary lifetime cost-per QALY black-box construct may not sit well with the CVS Health client base. Not only would exclusion decisions lack scientific credibility but they open the door, not only to challenges from manufacturers and their lobby groups with their own re-engineered models, but to likelihood of challenges also from patients, providers and their associations. At the same time, as emphasized above, establishing a technology assessment group would not only act as gatekeeper for new products but would be in a position for CVS Health monitor and challenge arbitrary pricing strategies over the lifetime of the product.

Conflicts of Interest: None declared

References 
${ }^{1}$ Langley PC. Resolving Lingering Problems or Continued Support for Pseudoscience? The ICER Value Assessment Update. Inov Pharm. 2017;8(4): No 7

${ }^{2}$ Langley PC. Transparency, Imaginary Worlds and ICER Value Assessments. Inov Pharm. 2017;8(4): No 11

${ }^{3}$ CVS Health. Current and new approaches to making drugs more affordable. August 2018.

${ }^{4}$ Langley PC. Validation of modeled pharmacoeconomic claims in formulary submissions. J Med Econ. 2015;18(12):993-99

${ }^{5}$ Langley PC. Sunlit uplands: the genius of the NICE reference case. Inov Pharm. 2016;7(2): No.12.

${ }^{6}$ Langley PC. Dreamtime: Version 5.0 of the Australian Guidelines for Preparing Submissions to the Pharmaceutical Benefits Advisory Committee (PBAC). Inov Pharm. 2017;8(1): No. 5

${ }^{7}$ Langley PC. True North: Building Imaginary Worlds with the Revised Canadian (CADTH) Guidelines for Health Technology Assessment. Inov Pharm. 2017;8(2): No. 9.

${ }^{8}$ Langley PC. Na domhain shamhlaíochta: formulary submission guidelines in Ireland and the standards of normal science. Curr Med Res Opin. 2016;32(5)

${ }^{9}$ Langley PC. He ao pohewa: The PHARMAC Prescription for Pharmacoeconomic Analysis in New Zealand and the standards of normal science. Inov Pharm. 2016;7(2): No. 13

${ }^{10}$ Canadian Agency for Drugs and Technologies in Health. Guidelines for the economic evaluation of health technologies: Canada $4^{\text {th }}$ Ed., Ottawa 2017

${ }^{11}$ Langley PC. True North: Building Imaginary Worlds with the Revised Canadian (CADTH) Guidelines for Health Technology Assessment. Inov Pharm. 2017;8(2): No. 9

${ }^{12}$ Langley PC. Cost-Effectiveness and Formulary Evaluation: Imaginary Worlds and Entresto Claims in Heart Failure. Inov Pharm. 2016;7(3): No. 6

${ }^{13}$ Langley PC. Multiple Sclerosis and the Comparative Value Disease Modifying Therapy Report of the Institute for Clinical and Economic Review (ICER). Inov Pharm. 2017;8(1): No. 12

${ }^{14}$ Langley PC. Imaginary Worlds and the Institute for Clinical and Economic Review (ICER) Evidence Report: Targeted Immune Modulators for Rheumatoid Arthritis. Inov Pharm. 2017;8(2): No. 10.

${ }^{15}$ Langley PC. Rush to Judgement: Imaginary Worlds and Cost-Outcomes Claims for PCSK9 Inhibitors. Inov Pharm. 2017;8(2): No. 11

${ }^{16}$ Langley PC. Alternative Facts and the ICER Proposed Policy on Access to Imaginary Pharmacoeconomic Worlds. Inov Pharm. 2018;9(2): No. 10

${ }^{17}$ Langley PC, Rhee TG. Imaginary Worlds: The status of simulation modeling in claims for cost-effectiveness in diabetes mellitus. Inov Pharm . 2016;7(2): No. 17

${ }^{18}$ Langley PC. Guidelines for Formulary Evaluation [Proposed]. Program in Social and Administrative Pharmacy. College of Pharmacy. University of Minnesota. Version 2.0. December 2016

${ }^{19}$ Langley PC. Nullius in Verba: Version 2.0 of the University of Minnesota, School of Social and Administrative Pharmacy Program, Proposed Guidelines for Formulary Evaluation. Inov Pharm. 2016;7(4): No 16

${ }^{20}$ Langley PC. Information or Evidence? Abandoning imaginary worlds for blockchains in health technology assessment. Inov Pharm. 2018;9(3): No. 12 
${ }^{21}$ Langley PC, Martin RE. Blockchains, property rights and health technology assessment in the pharmaceutical and device(s) industries. Inov Pharm. 2018;9(4): No. 1

22 Langley PC, Martin RE. Blockchains, formulary evaluations and health technology assessment. Inov Pharm. 2018;9(4): No. 2 